\title{
VALUE DISTRIBUTION OF THE SEQUENCES OF THE DERIVATIVES OF ITERATED POLYNOMIALS
}

\author{
Yûsuke Okuyama \\ Kyoto Institute of Technology, Division of Mathematics \\ Sakyo-ku, Kyoto 606-8585, Japan; okuyama@kit.ac.jp
}

\begin{abstract}
We establish the equidistribution of the sequence of the averaged pullbacks of a Dirac measure at any value in $\mathbf{C} \backslash\{0\}$ under the derivatives of the iterations of a polynomials $f \in \mathbf{C}[z]$ of degree more than one towards the $f$-equilibrium (or canonical) measure $\mu_{f}$ on $\mathbf{P}^{1}$. We also show that for every $C^{2}$ test function on $\mathbf{P}^{1}$, the convergence is exponentially fast up to a polar subset of exceptional values in C. A parameter space analog of the latter quantitative result for the monic and centered unicritical polynomials family is also established.
\end{abstract}

\section{Introduction}

Let $f \in \mathbf{C}[z]$ be a polynomial of degree $d>1$. Let $\mu_{f}$ be the $f$-equilibrium (or canonical) measure on $\mathbf{P}^{1}$, which coincides with the harmonic measure $\mu_{K(f)}$ on the filled-in Julia set $K(f)$ of $f$ with respect to $\infty$. The exceptional set $E(f):=\{a \in$ $\left.\mathbf{P}^{1}: \# \bigcup_{n \in \mathbf{N}} f^{-n}(a)<\infty\right\}$ of $f$ contains $\infty$ and $\# E(f) \leq 2$. Brolin [2, Theorem 16.1] studied the value distribution of the sequence $\left(f^{n}: \mathbf{P}^{1} \rightarrow \mathbf{P}^{1}\right)$ of the iterations of $f$, and established

$$
\left\{a \in \mathbf{P}^{1}: \lim _{n \rightarrow \infty} \frac{\left(f^{n}\right)^{*} \delta_{a}}{d^{n}}=\mu_{f} \text { weakly on } \mathbf{P}^{1}\right\}=\mathbf{P}^{1} \backslash E(f),
$$

which is more precise than the classical inclusion $\partial K(f) \subset \overline{\bigcup_{n \in \mathbf{N}} f^{-n}(a)}$ for every $a \in \mathbf{P}^{1} \backslash E(f)$. Here for every $h \in \mathbf{C}(z)$ of degree $>0$ and every Radon measure $\nu$ on $\mathbf{P}^{1}$, the pullback $h^{*} \nu$ of $\nu$ under $h$ is a Radon measure on $\mathbf{P}^{1}$ so that for every $a \in \mathbf{P}^{1}$, when $\nu=\delta_{a}, h^{*} \delta_{a}=\sum_{w \in h^{-1}(a)}\left(\operatorname{deg}_{w} h\right) \delta_{a}$ on $\mathbf{P}^{1}$. Pursuing the analogy between the roles played by $E(f)$ in (1.1) and by the set of Valiron exceptional values in $\mathbf{P}^{1}$ of a transcendental meromorphic function on C, Sodin [20], Russakovskii-Sodin [19], and Russakovskii-Shiffman [18] (see also [7], [15]) studied the value distribution of a sequence of rational maps between projective spaces from the viewpoint of Nevanlinna theory, in a quantitative way (cf. [22, Chapter V, §2]). Gauthier and Vigny [10, 1. in Theorem A] studied the value distribution of the sequence $\left(\left(f^{n}\right)^{\prime}: \mathbf{P}^{1} \rightarrow \mathbf{P}^{1}\right)$ of the derivatives of iterations of a polynomial $f \in \mathbf{C}[z]$ of degree $>1$ (cf. [23]) possibly with a polar subset of exceptional values in $\mathbf{C} \backslash\{0\}$, in terms of dynamics of the tangent map $F(z, w):=\left(f(z), f^{\prime}(z) w\right)$ on the tangent bundle TC. The aim of this article is to improve their result in two ways.

The first improvement of $[10,1$. in Theorem A] is qualitative, but with no exceptional values.

https://doi.org/10.5186/aasfm.2017.4233

2010 Mathematics Subject Classification: Primary 37F10; Secondary 30D35, 32H50.

Key words: Value distribution, equidistribution, quantitative equidistribution, derivative, iterated polynomials, monic and centered unicritical polynomials family, complex dynamics, Nevanlinna theory. 
Theorem 1. Let $f \in \mathbf{C}[z]$ be of degree $d>1$. Then for every $a \in \mathbf{C} \backslash\{0\}$,

$$
\lim _{n \rightarrow \infty} \frac{\left(\left(f^{n}\right)^{\prime}\right)^{*} \delta_{a}}{d^{n}-1}=\mu_{f}
$$

weakly on $\mathbf{P}^{1}$.

In Theorem 1 , the values $a=0, \infty$ are excluded since it is clear that for every $n \in \mathbf{N},\left(\left(f^{n}\right)^{\prime}\right)^{*} \delta_{\infty} /\left(d^{n}-1\right)=\delta_{\infty}\left(\neq \mu_{f}\right)$, and it immediately follows from (1.1) and the chain rule that $\lim _{n \rightarrow \infty}\left(\left(f^{n}\right)^{\prime} \delta_{0}\right) /\left(d^{n}-1\right)=\mu_{f}$ weakly on $\mathbf{P}^{1}$ if and only if $E(f)=\{\infty\}$. In Gauthier-Vigny [10,2. and 3. in Theorem A], they also established a result similar to Theorem 1 under the assumption that $f$ has no Siegel disks (or the assumption that $f$ is hyperbolic). Our proof of Theorem 1 is independent of their argument even in those cases.

The second improvement of $[10,1$. in Theorem $\mathrm{A}]$ is quantitative, but with an at most polar subset of exceptional values in $\mathbf{C}$.

Theorem 2. Let $f \in \mathbf{C}[z]$ be of degree $d>1$, and suppose that $E(f)=\{\infty\}$. Then for every $\eta>\sup _{z \in \mathbf{C}}$ : superattracting periodic point of $f \lim \sup _{n \rightarrow \infty}\left(\operatorname{deg}_{z}\left(f^{n}\right)\right)^{1 / n}$, there is a polar subset $E=E_{f, \eta}$ in $\mathbf{C}$ such that for every $a \in \mathbf{C} \backslash E$ and every $C^{2}$-test function $\phi$ on $\mathbf{P}^{1}$,

as $n \rightarrow \infty$.

$$
\int_{\mathbf{P}^{1}} \phi \mathrm{d}\left(\frac{\left(\left(f^{n}\right)^{\prime}\right)^{*} \delta_{a}}{d^{n}-1}-\mu_{f}\right)=o\left((\eta / d)^{n}\right)
$$

The proof of Theorem 2 is based on Russakovskii-Shiffman [18] mentioned above, and on an improvement of it for the sequence of the iterations of a rational function of degree $>1$ by Drasin and the author [6] (see also [4] and [21] in higher dimensions).

Remark 1.1. Under the assumption $E(f)=\{\infty\}$ in Theorem 2, we have $\sup _{z \in \mathbf{C} \text { : superattracting periodic point of } f} \limsup _{n \rightarrow \infty}\left(\operatorname{deg}_{z}\left(f^{n}\right)\right)^{1 / n} \in\{1,2, \ldots, d-1\}$, and $=1$ if and only if there is no superattracting cycles of $f$ in $\mathbf{C}$. Here we adopt the convention $\sup _{\emptyset}=1$. In the case that $E(f) \neq\{\infty\}$, we point out the following better estimate than that in Theorem 2

$$
\int_{\mathbf{P}^{1}} \phi \mathrm{d}\left(\frac{\left(\left(f^{n}\right)^{\prime}\right)^{*} \delta_{a}}{d^{n}-1}-\mu_{f}\right)=O\left(n d^{-n}\right) \quad \text { as } n \rightarrow \infty
$$

for every $a \in \mathbf{C} \backslash\{0\}$ and every $C^{2}$-test function $\phi$ on $\mathbf{P}^{1}$, with no exceptional values; indeed, we can assume that $f(z)=z^{d}$ without loss of generality (see Remark 3.1), and then $f^{n}(z)=z^{d^{n}}$ for every $n \in \mathbf{N}$ and $\mu_{f}$ is the normalized Lebesgue measure $m_{\partial \mathbf{D}}$ on the unit circle $\partial \mathbf{D}=\partial K(f)$. For every $a=r e^{i \theta}(r>0, \theta \in \mathbf{R})$, every $C^{1}$-test function $\phi$ on $\mathbf{P}^{1}$, and every $n \in \mathbf{N}$, we have $\mid \int_{\mathbf{P}^{1}} \phi \mathrm{d}\left(\left(\left(f^{n}\right)^{\prime}\right)^{*} \delta_{a}-\sum_{j=1}^{d^{n}-1} \delta_{\left.e^{i(\theta+j \cdot 2 \pi) /\left(d^{n}-1\right)}\right) /\left(d^{n}-\right.}\right.$ $1)\left|\leq\|\phi\|_{C^{1}} \cdot\right| e^{\left(\log \left(r d^{-n}\right)\right) /\left(d^{n}-1\right)}-1 \mid \leq\|\phi\|_{C^{1}} \cdot C n d^{-n}$ for some $C>0$ independent of both $\phi$ and $n$, and if $\phi$ is $C^{2}$, then by the midpoint method in numerically computing

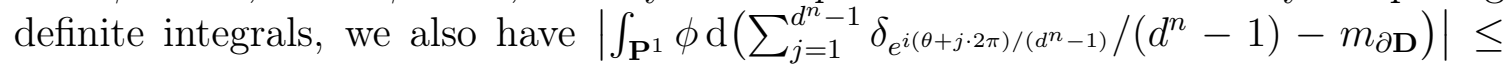
$\|\phi\|_{C^{2}} \cdot C^{\prime} d^{-n}$ for some $C^{\prime}>0$ independent of both $\phi$ and $n$.

Finally, let us focus on the (monic and centered) unicritical polynomials family

$$
f: \mathbf{C} \times \mathbf{P}^{1} \ni(\lambda, z) \mapsto z^{d}+\lambda=: f_{\lambda}(z) \in \mathbf{P}^{1}
$$

of degree $d>1$. The parameter space analog of Theorem 1 for the sequence $\left(\left(f_{\lambda}^{n}\right)^{\prime}(\lambda)\right)$ in $\mathbf{C}[\lambda]$ of the derivative of $f_{\lambda}^{n}$ at its unique critical value $z=\lambda$ in $\mathbf{C}$ is also obtained by Gauthier-Vigny [10, Theorem 3.7]. We will also establish a parameter space analog of Theorem 2. 
Theorem 3. Let $f$ be the monic and centered unicritical polynomials family of degree $d>1$ defined as in (1.2). Then for every $\eta>1$, there is a polar subset $E=E_{f, \eta}$ in $\mathbf{C}$ such that for every $a \in \mathbf{C} \backslash E$ and every $C^{2}$-test function $\phi$ on $\mathbf{P}^{1}$,

$$
\int_{\mathbf{P}^{1}} \phi(\lambda) \mathrm{d}\left(\frac{\left(\left(f_{\lambda}^{n}\right)^{\prime}(\lambda)\right)^{*} \delta_{a}}{d^{n}-1}-\mu_{C_{d}}\right)(\lambda)=O\left((\eta / d)^{n}\right)
$$

as $n \rightarrow \infty$. Here $C_{d}$ is the connectedness locus of the family $f$ in the parameter space $\mathrm{C}$ and $\mu_{C_{d}}$ is the harmonic measure on $C_{d}$ with pole $\infty$.

The proof of Theorem 3 is based on Russakovskii-Shiffman [18] mentioned above, and on a quantitative equidistribution of superattracting parameters by GauthierVigny [9].

In Section 2, we recall a background from complex dynamics. In Sections 3, 4, and 5 , we show Theorems 1,2 , and 3 , respectively.

Notation 1.2. We adopt the convention $\mathbf{N}=\mathbf{Z}_{>0}$. For every $a \in \mathbf{C}$ and every $r>0$, set $\mathbf{D}(a, r):=\{z \in \mathbf{C}:|z-a|<r\}$. Let $\delta_{z}$ be the Dirac measure on $\mathbf{P}^{1}$ at each $z \in \mathbf{P}^{1}$. Let $[z, w]$ be the chordal metric on $\mathbf{P}^{1}$ normalized as $[\cdot, \infty]=1 / \sqrt{1+|\cdot|^{2}}$ on $\mathbf{P}^{1}$ (following the notation in Nevanlinna's and Tsuji's books [14, 22]). Let $\omega$ be the Fubini-Study area element on $\mathbf{P}^{1}$ normalized as $\omega\left(\mathbf{P}^{1}\right)=1$. The Laplacian $\mathrm{dd}^{c}$ on $\mathbf{P}^{1}$ is normalized as $\operatorname{dd}^{c}(-\log [\cdot, \infty])=\omega-\delta_{\infty}$ on $\mathbf{P}^{1}$.

\section{Background}

2.1. Dynamics of rational functions. Let $f \in \mathbf{C}(z)$ be of degree $d>1$. Let $C(f)$ be the critical set of $f$. The Julia and Fatou sets of $f$ are defined by $J(f):=\left\{z \in \mathbf{P}^{1}\right.$ : the family $\left(f^{n}: \mathbf{P}^{1} \rightarrow \mathbf{P}^{1}\right)_{n \in \mathbf{N}}$ is not normal at $\left.z\right\}$ and $F(f):=$ $\mathbf{P}^{1} \backslash J(f)$, respectively. A component of $F(f)$ is called a Fatou component of $f$. A Fatou component $U$ of $f$ is mapped by $f$ properly onto a Fatou component of $f$. A Fatou component $U$ of $f$ is said to be cyclic if there is $n \in \mathbf{N}$ such that $f^{n}(U)=U$. For more details on complex dynamics, see e.g. Milnor's book [13].

The $f$-equilibrium (or canonical) measure $\mu_{f}$ on $\mathbf{P}^{1}$ is the unique probability Radon measure $\nu$ on $\mathbf{P}^{1}$ such that

$$
f^{*} \nu=d \cdot \nu \text { on } \mathbf{P}^{1}
$$

and that $\nu(\{a\})=0$ for every $a \in E(f)$; the exceptional set of $f$ is $E(f):=\{a \in$ $\left.\mathbf{P}^{1}: \# \bigcup_{n \in \mathbf{N}} f^{-n}(a)<\infty\right\}=\left\{a \in \mathbf{P}^{1}: f^{-2}(a)=\{a\}\right\}$. Then in fact $\operatorname{supp} \mu_{f}=J(f)$, and for every $n \in \mathbf{N}, \mu_{f^{n}}=\mu_{f}$ on $\mathbf{P}^{1}$. For more details, see Brolin [2], Lyubich [12], Freire-Lopes-Mañé [8].

2.2. Dynamics of polynomials. Let $f \in \mathbf{C}[z]$ be of degree $d>1$. We note that $\infty \in E(f), \#(C(f) \cap \mathbf{C}) \leq d-1$, and $C(f) \cap \mathbf{C}=\left(\operatorname{supp} \operatorname{dd}^{c} \log \left|f^{\prime}\right|\right) \cap \mathbf{C}$.

The filled-in Julia set $K(f)$ of $f$ is defined by

$$
K(f):=\left\{z \in \mathbf{C}: \limsup _{n \rightarrow \infty}\left|f^{n}(z)\right|<\infty\right\},
$$

whose complement in $\mathbf{P}^{1}$ coincides with the immediate superattractive basin

$$
I_{\infty}(f):=\left\{z \in \mathbf{P}^{1}: \lim _{n \rightarrow \infty} f^{n}(z)=\infty\right\}
$$

of the superattracting fixed point $\infty$ of $f$; in particular, $\lim _{n \rightarrow \infty} f^{n}=\infty$ locally uniformly on $I_{\infty}(f)$, and $K(f)$ is a compact subset in C. We note that $F(f)=$ $I_{\infty}(f) \cup \operatorname{int} K(f)$ and that $J(f)=\partial K(f)$. 
By a standard telescope argument, there exists the locally uniform limit

$$
g_{f}:=\lim _{n \rightarrow \infty} \frac{-\log \left[f^{n}(\cdot), \infty\right]}{d^{n}}
$$

on $\mathbf{C}$. Setting $g_{f}(\infty):=+\infty$, we have $g_{f} \circ f=d \cdot g_{f}$ on $\mathbf{P}^{1}$, and for every $n \in \mathbf{N}$, we also have $g_{f^{n}}=g_{f}$ on $\mathbf{P}^{1}$. The restriction of $g_{f}$ to $I_{\infty}(f)$ coincides with the Green function on $I_{\infty}(f)$ with pole $\infty$, and the measure

$$
\mu_{K(f)}:=\mathrm{dd}^{c} g_{f}+\delta_{\infty} \quad \text { on } \mathbf{P}^{1}
$$

coincides with the harmonic measure on $K(f)$ with pole $\infty$. In particular, supp $\mu_{K(f)}$ $\subset \partial K(f)$, and in fact $\mu_{K(f)}=\mu_{f}$ on $\mathbf{P}^{1}$. The function $z \mapsto g_{f}(z)-\log |z|$ extends harmonically to an open neighborhood of $\infty$ in $I_{\infty}(f)$ so the function $z \mapsto$ $-\log [z, \infty]-g_{f}(z)$ extends continuously to $\mathbf{P}^{1}$.

The following is substantially shown in Buff [3, the proof of Theorem 4].

Theorem 2.1. (Buff) Let $f \in \mathbf{C}[z]$ be of degree $d>1$, and let $z_{0} \in \mathbf{C}$. If $g_{f}\left(z_{0}\right) \geq \max _{c \in C(f) \cap \mathbf{C}} g_{f}(c)$, then $\left|f^{\prime}\left(z_{0}\right)\right| \leq d^{2} \cdot e^{(d-1) g_{f}\left(z_{0}\right)}$, and the equality never holds if $(C(f) \cap \mathbf{C}) \cap I_{\infty}(f) \neq \emptyset$.

For more details on polynomial dynamics and potential theory, see Brolin [2, Chapter III], and also Ransford's book [17].

\section{Proof of Theorem 1}

Let $f \in \mathbf{C}[z]$ be of degree $d>1$. For every $a \in \mathbf{C}$ and every $n \in \mathbf{N}$, the functions $\left(\log \left|\left(f^{n}\right)^{\prime}-a\right|\right) /\left(d^{n}-1\right)-g_{f}$ and $\left(\log \max \left\{1,\left|\left(f^{n}\right)^{\prime}\right|\right\}\right) /\left(d^{n}-1\right)-g_{f}$ extend continuously to $\mathbf{P}^{1}$. Set $a_{d}=a_{d}(f):=\lim _{n \rightarrow \infty} f(z) / z^{d} \in \mathbf{C} \backslash\{0\}$.

Remark 3.1. Since the question is affine invariant, we could assume $\left|a_{d}\right|=1$ without loss of generality, by replacing $f$ with $c^{-1} \circ f \circ c$ for such $c \in \mathbf{C} \backslash\{0\}$ that $c^{d-1}=a_{d}^{-1}$ if necessary (for every $c \in \mathbf{C} \backslash\{0\}, z \mapsto c \cdot z$ is also denoted by $c$ ). In this article, we would not normalize $f$ as $\left|a_{d}\right|=1$ in order to make it explicit which computations would be independent of such a normalization.

Lemma 3.2. On $I_{\infty}(f) \backslash \bigcup_{n \in \mathbf{N} \cup\{0\}} f^{-n}(C(f) \cap \mathbf{C})$,

$$
\lim _{n \rightarrow \infty}\left(\frac{\log \left|\left(f^{n}\right)^{\prime}\right|}{d^{n}-1}-g_{f}\right)=0
$$

locally uniformly.

Proof. For every $n \in \mathbf{N}$ and every $z \in \mathbf{C}$, by a direct calculation, we have

$$
\begin{aligned}
& \frac{\log \left|\left(f^{n}\right)^{\prime}(z)\right|}{d^{n}-1}-\frac{\log \left|d^{n} \cdot a_{d}^{\left(d^{n}-1\right) /(d-1)}\right|}{d^{n}-1}=\frac{1}{d^{n}-1} \int_{\mathbf{C}} \log |z-u|\left(\mathrm{dd}^{c} \log \left|\left(f^{n}\right)^{\prime}\right|\right)(u) \\
& =\frac{1}{d^{n}-1} \int_{\mathbf{C}} \sum_{j=0}^{n-1}\left(\int_{\mathbf{C}} \log |z-\cdot| \mathrm{d}\left(\left(f^{j}\right)^{*} \delta_{w}\right)\right)\left(\mathrm{dd}^{c} \log \left|f^{\prime}\right|\right)(w) \\
& =\frac{1}{d^{n}-1} \int_{\mathbf{C}} \sum_{j=0}^{n-1}\left(\log \left|f^{j}(z)-w\right|-\log \left|a_{d}\right|^{\left(d^{j}-1\right) /(d-1)}\right)\left(\operatorname{dd}^{c} \log \left|f^{\prime}\right|\right)(w) \\
& =\frac{1}{d^{n}-1} \int_{\mathbf{C}} \sum_{j=0}^{n-1}\left(\log \left[f^{j}(z), w\right]-\log \left[f^{j}(z), \infty\right]-\log [w, \infty]\right)\left(\operatorname{dd}^{c} \log \left|f^{\prime}\right|\right)(w) \\
& \quad-\log \left|a_{d}\right|^{\frac{1}{d-1}-\frac{n}{d^{n}-1}}
\end{aligned}
$$


Then noting that $g_{f} \circ f=d \cdot g_{f}$ on $\mathbf{P}^{1}$, for every $n \in \mathbf{N}$ and every $z \in \mathbf{P}^{1}$, we have

$$
\begin{aligned}
& \frac{\log \left|\left(f^{n}\right)^{\prime}(z)\right|}{d^{n}-1}-g_{f}(z)=\frac{1}{d^{n}-1} \int_{\mathbf{C}}\left(\sum_{j=0}^{n-1} \log \left[f^{j}(z), w\right]\right)\left(\mathrm{dd}^{c} \log \left|f^{\prime}\right|\right)(w) \\
& +\frac{d-1}{d^{n}-1} \sum_{j=0}^{n-1}\left(-\log \left[f^{j}(z), \infty\right]-g_{f}\left(f^{j}(z)\right)\right) \\
& \quad+\left(-\int_{\mathbf{C}} \log [w, \infty]\left(\mathrm{dd}^{c} \log \left|f^{\prime}\right|\right)(w)+\log d+\log \left|a_{d}\right|\right) \frac{n}{d^{n}-1},
\end{aligned}
$$

which with $\sup _{z \in \mathbf{P}^{1}}\left|-\log [z, \infty]-g_{f}(z)\right|<\infty$ completes the proof.

Lemma 3.3. There is $C=C_{f}>0$ such that for every $n \in \mathbf{N}$ and every $z \in \mathbf{P}^{1}$,

$$
\frac{\log \max \left\{1,\left|\left(f^{n}\right)^{\prime}(z)\right|\right\}}{d^{n}-1}-g_{f}(z) \leq \frac{C n}{d^{n}-1} .
$$

Proof. Set

$$
\begin{aligned}
C=C_{f}:= & (d-1) \cdot \sup _{z \in \mathbf{P}^{1}}\left|-\log [z, \infty]-g_{f}(z)\right| \\
& +(d-1) \cdot \sup _{w \in C(f) \cap \mathbf{C}}|\log [w, \infty]|+\log d+|\log | a_{d}|| \in \mathbf{R}_{>0} .
\end{aligned}
$$

Then for every $n \in \mathbf{N}$ and every $z \in \mathbf{C}$, from (3.2), we have $\left|\left(f^{n}\right)^{\prime}(z)\right| \leq e^{C n}$. $e^{\left(d^{n}-1\right) g_{f}(z)}$, which with $g_{f} \geq 0$ on $\mathbf{P}^{1}$ completes the proof.

We note that $\max _{c \in \bigcup_{n \in \mathrm{N} \cup\{0\}} f^{-n}(C(f) \cap \mathbf{C})} g_{f}(c)=\max _{c \in C(f) \cap \mathbf{C}} g_{f}(c)<\infty$ by $g_{f} \circ f=$ $d \cdot g_{f}$ on $\mathbf{P}^{1}$.

Lemma 3.4. For every $a \in \mathbf{C} \backslash\{0\}$,

$$
\lim _{n \rightarrow \infty} \int_{\mathbf{P}^{1}}\left|\frac{\log \left|\left(f^{n}\right)^{\prime}-a\right|}{d^{n}-1}-g_{f}\right| \mathrm{d} \omega=0
$$

Proof. Fix $a \in \mathbf{C} \backslash\{0\}$. The sequence $\left(\left(\log \left|\left(f^{n}\right)^{\prime}-a\right|\right) /\left(d^{n}-1\right)\right)$ of subharmonic functions on $\mathbf{C}$ is locally uniformly bounded from above on $\mathbf{C}$; indeed, by the chain rule and $\liminf _{z \rightarrow \infty}\left|f^{\prime}(z)\right|=+\infty$, for every $R>0$ so large that $\{|z|=R\} \subset I_{\infty}(f) \backslash \bigcup_{n \in \mathbf{N} \cup\{0\}} f^{-n}(C(f) \cap \mathbf{C})$, we have $\liminf _{n \rightarrow \infty} \inf _{|z|=R}\left|\left(f^{n}\right)^{\prime}(z)\right|=$ $+\infty$, which with the maximum modulus principle yields $\sup _{|z| \leq R}\left|\left(f^{n}\right)^{\prime}(z)-a\right| \leq$ $\sup _{|z|=R} 2\left|\left(f^{n}\right)^{\prime}(z)\right|$ for every $n \in \mathbf{N}$ large enough. Then by Lemma 3.3, we have $\lim \sup _{n \rightarrow \infty} \sup _{|z| \leq R}\left(\log \left|\left(f^{n}\right)^{\prime}-a\right|\right) /\left(d^{n}-1\right) \leq \sup _{|z|=R} g_{f}(z)<\infty$. By Lemma 3.2 and $g_{f}>0$ on $I_{\infty}(f)$, for every compact subset $C$ in $I_{\infty}(f) \backslash \bigcup_{n \in \mathbf{N} \cup\{0\}} f^{-n}(C(f) \cap \mathbf{C})$, we also have $1 / 2 \leq\left|\left(\left(f^{n}\right)^{\prime}-a\right) /\left(f^{n}\right)^{\prime}\right| \leq 2$ on $C$ for every $n \in \mathbf{N}$ large enough, so in particular

$$
\lim _{n \rightarrow \infty}\left(\frac{\log \left|\left(f^{n}\right)^{\prime}-a\right|}{d^{n}-1}-g_{f}\right)=\lim _{n \rightarrow \infty}\left(\frac{\log \left|\left(f^{n}\right)^{\prime}\right|}{d^{n}-1}-g_{f}\right)=0
$$

locally uniformly on $I_{\infty}(f) \backslash \bigcup_{n \in \mathbf{N} \cup\{0\}} f^{-n}(C(f) \cap \mathbf{C})$.

Let $m_{2}$ be the Lebesgue measure on $\mathbf{C}$. By a compactness principle for a locally uniformly upper bounded sequence of subharmonic functions on a domain in $\mathbf{R}^{m}$ which is not locally uniformly convergent to $-\infty$ (see Azarin [1, Theorem 1.1.1], Hörmander's book [11, Theorem 4.1.9(a)]), we can choose a sequence $\left(n_{j}\right)$ in $\mathbf{N}$ tending to $+\infty$ as $j \rightarrow \infty$ such that the $L_{\text {loc }}^{1}\left(\mathbf{C}, m_{2}\right)$-limit $\phi:=\lim _{j \rightarrow \infty}\left(\log \mid\left(f^{n_{j}}\right)^{\prime}-\right.$ $a \mid) /\left(d^{n_{j}}-1\right)$ exists and is subharmonic on $\mathbf{C}$. Choosing a subsequence of $\left(n_{j}\right)$ if 
necessary, we have $\phi=\lim _{j \rightarrow \infty}\left(\log \left|\left(f^{n_{j}}\right)^{\prime}-a\right|\right) /\left(d^{n_{j}}-1\right)$ Lebesgue a.e. on C. Then by (3.5), we have $\phi \equiv g_{f}$ Lebesgue a.e. on $\mathbf{C} \backslash\left(K(f) \cup \bigcup_{n \in \mathbf{N} \cup\{0\}} f^{-n}(C(f) \cap \mathbf{C})\right)$, and in turn on $\mathbf{C} \backslash K(f)$ by the subharmonicity of $\phi$ and the harmonicity of $g_{f}$ there. Let us show that $\phi=g_{f}$ Lebesgue a.e. on the whole $\mathbf{C}$, and then $\lim _{n \rightarrow \infty}\left(\log \mid\left(f^{n}\right)^{\prime}-\right.$ $a \mid) /\left(d^{n}-1\right)=g_{f}$ in $L_{\mathrm{loc}}^{1}\left(\mathbf{C}, m_{2}\right)$, which with the locally uniform convergence (3.5) will complete the proof since $\max _{c \in \cup_{n \in \mathbf{N} \cup\{0\}} f^{-n}(C(f) \cap \mathbf{C})} g_{f}(c)<\infty$ and the RadonNikodym derivative $\mathrm{d} \omega / \mathrm{d} m_{2}$ is continuous so locally bounded on $\mathbf{C}$.

By $\log (1 /[w, \infty])-\log \max \{1,|w|\}) \leq \log \sqrt{2}$ on $\mathbf{C}$ and Lemma 3.3, for every $n \in \mathbf{N}$, we have

$$
\begin{aligned}
\frac{\log \left|\left(f^{n}\right)^{\prime}-a\right|}{d^{n}-1}-g_{f} & =\frac{\log \left[\left(f^{n}\right)^{\prime}, a\right]}{d^{n}-1}+\left(\frac{\log \left(1 /\left[\left(f^{n}\right)^{\prime}, \infty\right]\right)}{d^{n}-1}-g_{f}\right)+\frac{\log (1 /[a, \infty])}{d^{n}-1} \\
& \leq \frac{C_{f} \cdot n}{d^{n}-1}+\frac{\log \sqrt{2}+\log (1 /[a, \infty])}{d^{n}-1}
\end{aligned}
$$

on $\mathbf{C}$, so $\phi \leq g_{f}$ Lebesgue a.e. on $\mathbf{C}$ and in turn on $\mathbf{C}$ by the subharmonicity of $\phi$ and the continuity of $g_{f}$ on $\mathbf{C}$. Hence $\phi-g_{f}$ is $\leq 0$ and is upper semicontinuous on C.

Now suppose to the contrary that the open subset $\left\{z \in \mathbf{C}: \phi(z)<g_{f}(z)\right\}$ in $\mathbf{C}$ is non-empty. Then by $\phi \equiv g_{f}$ on $\mathbf{C} \backslash K(f)$, there is a bounded Fatou component $U$ of $f$ containing a component $W$ of $\left\{z \in \mathbf{C}: \phi(z)<g_{f}(z)\right\}$. Since $\phi \leq g_{f}=0$ on $U \subset K(f)$, by the maximum principle for subharmonic functions, we in fact have $U=W$.

Taking a subsequence of $\left(n_{j}\right)$ if necessary, we can assume that $\left(f^{n_{j}} \mid U\right)$ is locally uniformly convergent to a holomorphic function $g$ on $U$ as $j \rightarrow \infty$ without loss of generality. We claim that $g^{\prime} \equiv a$ on $U$, so we can say $g \in \mathbf{C}[z]$; indeed, fixing a domain $D \Subset U=W$, by a version of Hartogs's lemma on subharmonic functions (see Hörmander's book [11, Theorem 4.1.9(b)]) and the upper semicontinuity of $\phi$, we have $\lim \sup _{n \rightarrow \infty} \sup _{\bar{D}}\left(\log \left|\left(f^{n_{j}}\right)^{\prime}-a\right|\right) /\left(d^{n_{j}}-1\right) \leq \sup _{\bar{D}} \phi<0$. Hence $g^{\prime}=$ $\left(\lim _{j \rightarrow \infty} f^{n_{j}}\right)^{\prime}=\lim _{j \rightarrow \infty}\left(f^{n_{j}}\right)^{\prime} \equiv a$ on $D$, so $g^{\prime} \equiv a$ on $U$ by the identity theorem for holomorphic functions.

Hence, under the assumption that $a \neq 0$, the locally uniform limit $g$ on $U$ is nonconstant. So by Hurwitz's theorem and the classification of cyclic Fatou components, there is $N \in \mathbf{N}$ such that $V:=f^{n_{N}}(U)=g(U)(\supset g(\bar{D}))$ is a Siegel disk of $f$ and, setting $p:=\min \left\{n \in \mathbf{N}: f^{n}(V)=V\right\}$, that $p \mid\left(n_{j}-n_{N}\right)$ for every $j \geq N$. We can fix a holomorphic injection $h: V \rightarrow \mathbf{C}$ such that for some $\alpha \in \mathbf{R} \backslash \mathbf{Q}$, setting $\lambda:=e^{2 i \pi \alpha}$, we have $h \circ f^{p}=\lambda \cdot h$ on $V$, so for every $j \geq N, h \circ f^{n_{j}}=\lambda^{\left(n_{j}-n_{N}\right) / p} \cdot\left(h \circ f^{n_{N}}\right)$ on $U$. Then taking a subsequence of $\left(n_{j}\right)$ if necessary, there also exists the limit

$$
\lambda_{0}:=\lim _{j \rightarrow \infty} \lambda^{\left(n_{j}-n_{N}\right) / p}
$$

in $\partial \mathbf{D}$, so that $h \circ g=\lim _{j \rightarrow \infty} h \circ f^{n_{j}}=\lambda_{0} \cdot\left(h \circ f^{n_{N}}\right)$ on $U$. In particular,

$$
h \circ f^{n_{j}}-h \circ g=\left(\lambda^{\left(n_{j}-n_{N}\right) / p}-\lambda_{0}\right) \cdot\left(h \circ f^{n_{N}}\right)
$$

on $U$. Set $w_{0}:=h^{-1}(0) \in V$, so that $f^{p}\left(w_{0}\right)=w_{0}$, and fix $z_{0} \in f^{-n_{N}}\left(w_{0}\right) \cap U$, so that $f^{n_{j}}\left(z_{0}\right)=w_{0}$ for every $j \geq N$ and $g\left(z_{0}\right)=\lim _{j \rightarrow \infty} f^{n_{j}}\left(z_{0}\right)=w_{0}$.

We claim that

$$
\frac{\log \left|\left(f^{n_{j}}\right)^{\prime}\left(z_{0}\right)-a\right|}{d^{n_{j}}-1}=\frac{\log \left|\lambda^{\left(n_{j}-n_{N}\right) / p}-\lambda_{0}\right|}{d^{n_{j}}-1}+O\left(d^{-n_{j}}\right)
$$


as $j \rightarrow \infty$; for, by the chain rule applied to both sides in (3.6) and $h^{\prime}\left(w_{0}\right) \neq 0$ (and $\left.g^{\prime}\left(z_{0}\right)=a\right)$, we have

$$
\left(f^{n_{j}}\right)^{\prime}\left(z_{0}\right)-a=\left(\lambda^{\left(n_{j}-n_{N}\right) / p}-\lambda_{0}\right) \cdot\left(f^{n_{N}}\right)^{\prime}\left(z_{0}\right),
$$

which also yields $\left(f^{n_{N}}\right)^{\prime}\left(z_{0}\right) \neq 0$ by $\left(f^{n_{j}}\right)^{\prime}\left(z_{0}\right)=\left(f^{n_{j}-n_{N}}\right)^{\prime}\left(w_{0}\right) \cdot\left(f^{n_{N}}\right)^{\prime}\left(z_{0}\right)$ and the assumption $a \neq 0$. We also claim that

$$
\liminf _{j \rightarrow \infty} \frac{1}{d^{n_{j}}} \log \left|\lambda^{\left(n_{j}-n_{N}\right) / p}-\lambda_{0}\right| \geq 0
$$

(cf. [16, Proof of Theorem 3]); indeed, for every domain $D \Subset U \backslash f^{-n_{N}}\left(w_{0}\right)$, since $h^{-1}$ is Lipschitz continuous on $\left.h\left(\bigcup_{n \in \mathbf{N}}\left(f^{p}\right)^{n}\left(f^{n_{N}}(D)\right)\right) \cup g(D)\right) \Subset h(V)$ and $\sup _{D}\left|h \circ f^{n_{N}}\right|>$ 0 , from (3.6), we observe that

$$
\frac{1}{d^{n_{j}}} \sup _{D} \log \left|f^{n_{j}}-g\right| \leq \frac{1}{d^{n_{j}}} \log \left|\lambda^{\left(n_{j}-n_{N}\right) / p}-\lambda_{0}\right|+O\left(d^{-n_{j}}\right)
$$

as $j \rightarrow \infty$. On the other hand, for every domain $\tilde{D}$ intersecting $\partial U$ in $\mathbf{C}$, fixing $\tilde{z} \in \tilde{D} \cap I_{\infty}(f) \neq \emptyset$, we observe that

$$
\liminf _{j \rightarrow \infty} \frac{1}{d^{n_{j}}} \sup _{\tilde{D}} \log \left|f^{n_{j}}-g\right| \geq g_{f}(\tilde{z})>0 .
$$

Now fix $z_{1} \in U$ and $z^{\prime} \in \partial U$ such that $\mathbf{D}\left(z_{1},\left|z^{\prime}-z_{1}\right|\right) \subset U \backslash f^{-n_{N}}\left(w_{0}\right)$. Then for every $\epsilon \in\left(0,\left|z^{\prime}-z_{1}\right|\right)$, using Cauchy's estimate applied to $f^{n_{j}}-g \in \mathbf{C}[z]$ around $z_{1}$, we have

$$
\begin{aligned}
\left|f^{n_{j}}-g\right| & \leq \sum_{k=0}^{d^{n_{j}}} \frac{\sup _{\partial \mathbf{D}\left(z_{1},\left|z^{\prime}-z_{1}\right|-\epsilon\right)}\left|f^{n_{j}}-g\right|}{\left(\left|z^{\prime}-z_{1}\right|-\epsilon\right)^{k}}\left|\cdot-z_{1}\right|^{k} \\
& \leq\left(\sup _{\mathbf{D}\left(z_{1},\left|z^{\prime}-z_{1}\right|-\epsilon\right)}\left|f^{n_{j}}-g\right|\right) \cdot \sum_{k=0}^{d^{n_{j}}}\left(\frac{\left|z^{\prime}-z_{1}\right|+\epsilon}{\left|z^{\prime}-z_{1}\right|-\epsilon}\right)^{k}
\end{aligned}
$$

on $\mathbf{D}\left(z^{\prime}, \epsilon\right)$, so since $z^{\prime} \in \mathbf{D}\left(z^{\prime}, \epsilon\right) \cap \partial U$ and $\mathbf{D}\left(z_{1},\left|z^{\prime}-z_{1}\right|-\epsilon\right) \Subset U \backslash f^{-n_{N}}\left(w_{0}\right)$, by $\left(*^{*}\right)$ and $(*)$, we have

$$
\begin{aligned}
0 & <\left(\liminf _{j \rightarrow \infty} \frac{1}{d^{n_{j}}} \log \sup _{\mathbf{D}\left(z^{\prime}, \epsilon\right)}\left|f^{n_{j}}-g\right|\right. \\
& \leq \liminf _{j \rightarrow \infty} \frac{1}{d^{n_{j}}} \log \sup _{\mathbf{D}\left(z_{1},\left|z^{\prime}-z_{1}\right|-\epsilon\right)}\left|f^{n_{j}}-g\right|+\log \frac{\left|z^{\prime}-z_{1}\right|+\epsilon}{\left|z^{\prime}-z_{1}\right|-\epsilon} \\
& \leq) \liminf _{j \rightarrow \infty} \frac{1}{d^{n_{j}}} \log \left|\lambda^{\left(n_{j}-n_{N}\right) / p}-\lambda_{0}\right|+\log \frac{\left|z^{\prime}-z_{1}\right|+\epsilon}{\left|z^{\prime}-z_{1}\right|-\epsilon} .
\end{aligned}
$$

This yields (3.8) as $\epsilon \rightarrow 0$.

Once (3.7) and (3.8) are at our disposal, using a version of Hartogs's lemma on subharmonic functions again, we have

$$
\phi\left(z_{0}\right) \geq \limsup _{j \rightarrow \infty} \frac{\log \left|\left(f^{n_{j}}\right)^{\prime}\left(z_{0}\right)-a\right|}{d^{n_{j}}-1} \geq \liminf _{j \rightarrow \infty} \frac{\log \left|\lambda^{\left(n_{j}-n_{N}\right) / p}-\lambda_{0}\right|}{d^{n_{j}}-1} \geq 0,
$$

which contradicts $\phi<g_{f}=0$ on $U=W$. 
For every $a \in \mathbf{C} \backslash\{0\}$ and every $C^{2}$-test function $\phi$ on $\mathbf{P}^{1}$, by Lemma 3.4, we have

$$
\begin{aligned}
& \left|\int_{\mathbf{P}^{1}} \phi \mathrm{d}\left(\frac{\left(\left(f^{n}\right)^{\prime}\right)^{*} \delta_{a}}{d^{n}-1}-\mu_{f}\right)\right|=\left|\int_{\mathbf{P}^{1}} \phi \mathrm{dd}^{c}\left(\frac{\log \left|\left(f^{n}\right)^{\prime}(\cdot)-a\right|}{d^{n}-1}-g_{f}\right)\right| \\
& \leq\left(\sup _{\mathbf{P}^{1}}\left|\frac{\mathrm{dd}^{c} \phi}{\mathrm{d} \omega}\right|\right) \cdot \int_{\mathbf{P}^{1}}\left|\frac{\log \left|\left(f^{n}\right)^{\prime}(z)-a\right|}{d^{n}-1}-g_{f}\right| \mathrm{d} \omega(z) \rightarrow 0 \quad \text { as } n \rightarrow \infty,
\end{aligned}
$$

where the Radon-Nikodym derivative $\left(\mathrm{dd}^{c} \phi\right) / \mathrm{d} \omega$ on $\mathbf{P}^{1}$ is bounded on $\mathbf{P}^{1}$.

\section{Proof of Theorem 2}

Let $f \in \mathbf{C}[z]$ be of degree $d>1$, and suppose that $E(f)=\{\infty\}$. Then

$$
\begin{aligned}
& \sup _{z \in \mathbf{C}: \text { superattracting periodic point of } f} \limsup _{n \rightarrow \infty}\left(\operatorname{deg}_{z}\left(f^{n}\right)\right)^{1 / n} \\
& =\sup _{c \in C(f) \cap \mathbf{C}: \text { periodic under } f} \limsup _{n \rightarrow \infty}\left(\operatorname{deg}_{c}\left(f^{n}\right)\right)^{1 / n} \in\{1,2, \ldots, d-1\}
\end{aligned}
$$

(recall the convention $\sup _{\emptyset}=1$ ). Set $a_{d}:=a_{d}(f)=\lim _{n \rightarrow \infty} f(z) / z^{d} \in \mathbf{C} \backslash\{0\}$. For every $n \in \mathbf{N}$, the functions $\left(\log \left(1 /\left[\left(f^{n}\right)^{\prime}, \infty\right]\right) /\left(d^{n}-1\right)-g_{f}\right.$ and $\left(\log \max \left\{1,\left|\left(f^{n}\right)^{\prime}\right|\right\}\right) /\left(d^{n}-\right.$ 1) $-g_{f}$ extend continuously to $\mathbf{P}^{1}$.

Lemma 4.1. For every $\eta>\sup _{c \in C(f) \cap C}$ : periodic under $f \lim \sup _{n \rightarrow \infty}\left(\operatorname{deg}_{c}\left(f^{n}\right)\right)^{1 / n}$,

$$
\int_{\mathbf{P}^{1}}\left|\frac{\log \left(1 /\left[\left(f^{n}\right)^{\prime}, \infty\right]\right)}{d^{n}-1}-g_{f}\right| \mathrm{d} \omega=o\left((\eta / d)^{n}\right)
$$

as $n \rightarrow \infty$.

Proof. For every $n \in \mathbf{N}$, from (3.2), we have

$$
\begin{aligned}
& \int_{\mathbf{P}^{1}}\left|\frac{\log \left|\left(f^{n}\right)^{\prime}(z)\right|}{d^{n}-1}-g_{f}(z)\right| \mathrm{d} \omega(z) \\
& \leq \frac{1}{d^{n}-1} \int_{\mathbf{C}}\left(\sum_{j=0}^{n-1} \int_{\mathbf{P}^{1}} \log \frac{1}{\left[f^{j}(z), w\right]} \mathrm{d} \omega(z)\right)\left(\operatorname{dd}^{c} \log \left|f^{\prime}\right|\right)(w)+\frac{C_{f} \cdot n}{d^{n}-1},
\end{aligned}
$$

where $C_{f}>0$ is defined in (3.4). By [6, Theorem 2], for every $\eta>\sup _{c \in C(f) \cap C \text { : periodic }}$ under $f \lim \sup _{n \rightarrow \infty}\left(\operatorname{deg}_{c}\left(f^{n}\right)\right)^{1 / n}$ and every $w \in \mathbf{C}\left(=\mathbf{P}^{1} \backslash E(f)\right.$ under the assumption $E(f)=\{\infty\})$, we have

$$
\int_{\mathbf{P}^{1}} \log \frac{1}{\left[f^{n}(z), w\right]} \mathrm{d} \omega(z)=o\left(\eta^{n}\right)
$$

as $n \rightarrow \infty$, which with Lemma 3.3 and $0 \leq \log (1 /[w, \infty])-\log \max \{1,|w|\} \leq \log \sqrt{2}$ on $\mathbf{C}$ completes the proof.

Lemma 4.2. For every $\eta>1$, the Valiron exceptional set

$$
E_{V}\left(\left(\left(f^{n}\right)^{\prime}\right),\left(\eta^{n}\right)\right):=\left\{a \in \mathbf{P}^{1}: \limsup _{n \rightarrow \infty} \frac{1}{\eta^{n}} \int_{\mathbf{P}^{1}} \log \frac{1}{\left[\left(f^{n}\right)^{\prime}(z), a\right]} \mathrm{d} \omega(z)>0\right\}
$$

of the sequence $\left(\left(f^{n}\right)^{\prime}\right)$ of the derivatives of the iterations of $f$ with respect to the sequence $\left(\eta^{n}\right)$ in $\mathbf{R}_{>0}$ is a polar subset in $\mathbf{P}^{1}$. 
Proof. This is an application of Russakovskii-Shiffman [18, Proposition 6.2] to the sequence $\left(\left(f^{n}\right)^{\prime}\right)$ in $\mathbf{C}[z]$ since $\sum_{n \in \mathbf{N}} 1 / \eta^{n}<\infty$ for every $\eta>1$.

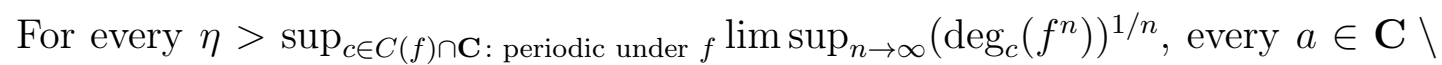
$E_{V}\left(\left(\left(f^{n}\right)^{\prime}\right),\left(\eta^{n}\right)\right)$, and every $C^{2}$-test function $\phi$ on $\mathbf{P}^{1}$, by Lemmas 4.1 and 4.2 , we have

$$
\begin{aligned}
\left|\int_{\mathbf{P}^{1}} \phi \mathrm{d}\left(\frac{\left(\left(f^{n}\right)^{\prime}\right)^{*} \delta_{a}}{d^{n}-1}-\mu_{f}\right)\right|= & \left|\int_{\mathbf{P}^{1}} \phi \operatorname{dd}^{c}\left(\frac{\log \left[\left(f^{n}\right)^{\prime}, a\right]}{d^{n}-1}+\frac{\log \left(1 /\left[\left(f^{n}\right)^{\prime}, \infty\right]\right)}{d^{n}-1}-g_{f}\right)\right| \\
\leq & \left.\left(\sup _{\mathbf{P}^{1}} \mid \frac{\mathrm{dd}^{c} \phi}{\mathrm{d} \omega}\right)\right)\left(\frac{1}{d^{n}-1} \int_{\mathbf{P}^{1}} \log \frac{1}{\left[\left(f^{n}\right)^{\prime}(z), a\right]} \mathrm{d} \omega(z)\right. \\
& \left.+\int_{\mathbf{P}^{1}}\left|\frac{\log \left(1 /\left[\left(f^{n}\right)^{\prime}(z), \infty\right]\right)}{d^{n}-1}-g_{f}\right| \mathrm{d} \omega(z)\right) \\
= & o\left((\eta / d)^{n}\right) \text { as } n \rightarrow \infty
\end{aligned}
$$

where the Radon-Nikodym derivative $\left(\mathrm{dd}^{c} \phi\right) / \mathrm{d} \omega$ on $\mathbf{P}^{1}$ is bounded on $\mathbf{P}^{1}$.

\section{Proof of Theorem 3}

Let $f: \mathbf{C} \times \mathbf{P}^{1} \ni(\lambda, z) \mapsto z^{d}+\lambda=: f_{\lambda}(z) \in \mathbf{P}^{1}$ be the monic and centered unicritical polynomials family of degree $d>1$. For every $n \in \mathbf{N}, f_{\lambda}^{n}(\lambda),\left(f_{\lambda}^{n}\right)^{\prime}(\lambda) \in$ $\mathbf{C}[\lambda]$ are of degree $d^{n}, d^{n}-1$, respectively.

5.1. Background on the family $\boldsymbol{f}$. Recall the definitions in Subsection 2.2. The following constructions are due to Douady-Hubbard [5] and Sibony.

For every $\lambda \in \mathbf{C}, f_{\lambda}^{\prime}(z)=d \cdot z^{d-1}$, so $C\left(f_{\lambda}\right) \cap \mathbf{C}=\{0\}$ and $f_{\lambda}(0)=\lambda$. The connectedness locus $C_{d}:=\left\{\lambda \in \mathbf{C}: \lambda \in K\left(f_{\lambda}\right)\right\}$ of the family $f$ is a compact subset in $\mathbf{C}$, and $H_{\infty}=H_{d, \infty}:=\mathbf{P}^{1} \backslash C_{d}$ is a simply connected domain containing $\infty$ in $\mathbf{P}^{1}$. Moreover, the locally uniform limit

$$
g_{H_{\infty}}(\lambda):=g_{f_{\lambda}}(\lambda)=d \cdot g_{f_{\lambda}}(0)=\lim _{n \rightarrow \infty} \frac{-\log \left[f_{\lambda}^{n}(\lambda), \infty\right]}{d^{n}}
$$

exists on C. Setting $g_{H_{\infty}}(\infty):=+\infty$, the restriction of $g_{H_{\infty}}$ to $H_{\infty}$ coincides with the Green function on $H_{\infty}$ with pole $\infty$, and the measure

$$
\mu_{C_{d}}:=\operatorname{dd}^{c} g_{H_{\infty}}+\delta_{\infty} \quad \text { on } \mathbf{P}^{1}
$$

coincides with the harmonic measure on $C_{d}$ with pole $\infty$. In particular, $z \mapsto g_{H_{\infty}}(z)-$ $\log |z|$ extends harmonically to an open neighborhood of $\infty$ in $H_{\infty}$, and $\operatorname{supp} \mu_{C_{d}} \subset$ $\partial C_{d}$ (in fact, the equality holds).

5.2. Proof of Theorem 3. For every $n \in \mathbf{N}, \lambda \mapsto\left(\log \left|\left(f_{\lambda}^{n}\right)^{\prime}(\lambda)\right|\right) /\left(d^{n}-1\right)-$ $g_{H_{\infty}}(\lambda)$ and $\lambda \mapsto\left(\log \max \left\{1,\left|\left(f_{\lambda}^{n}\right)^{\prime}(\lambda)\right|\right\}\right) /\left(d^{n}-1\right)-g_{H_{\infty}}(\lambda)$ on $\mathbf{C}$ extend continuously to $\mathbf{P}^{1}$.

Lemma 5.1. For every $n \in \mathbf{N}$ and every $\lambda \in \mathbf{C}$,

$$
\frac{\log \max \left\{1,\left|\left(f_{\lambda}^{n}\right)^{\prime}(\lambda)\right|\right\}}{d^{n}-1}-g_{H_{\infty}}(\lambda) \leq \frac{n \log \left(d^{2}\right)}{d^{n}-1} .
$$

Proof. For every $n \in \mathbf{N}$ and every $\lambda \in \mathbf{C}$, by $g_{f_{\lambda}^{n}}=g_{f_{\lambda}}$ on $\mathbf{P}^{1}$ and $g_{f_{\lambda}} \circ f_{\lambda}=d \cdot g_{f_{\lambda}}$ on $\mathbf{P}^{1}$, we have $g_{f_{\lambda}^{n}}(\lambda)=g_{f_{\lambda}}(\lambda)=d \cdot g_{f_{\lambda}}(0) \geq g_{f_{\lambda}}(0)=\max _{c \in C\left(f_{\lambda}\right) \cap \mathbf{C}} g_{f_{\lambda}}(c)=$ $\max _{c \in C\left(f_{\lambda}^{n}\right) \cap \mathbf{C}} g_{f_{\lambda}^{n}}(c)$, so by Theorem 2.1, we have $\left|\left(f_{\lambda}^{n}\right)^{\prime}(\lambda)\right| \leq\left(d^{n}\right)^{2} e^{\left(d^{n}-1\right) g_{f_{\lambda}^{n}}(\lambda)}=$ $\left(d^{n}\right)^{2} e^{\left(d^{n}-1\right) g_{f_{\lambda}}(\lambda)}=\left(d^{n}\right)^{2} e^{\left(d^{n}-1\right) g_{H_{\infty}}(\lambda)}$. This with $g_{H_{\infty}}(\lambda) \geq 0$ completes the proof. 


\section{Lemma 5.2.}

$$
\int_{\mathbf{P}^{1}}\left|\frac{\log \left(1 /\left[\left(f_{\lambda}^{n}\right)^{\prime}(\lambda), \infty\right]\right)}{d^{n}-1}-g_{H_{\infty}}(\lambda)\right| \mathrm{d} \omega(\lambda)=O\left(n^{2} d^{-n}\right)
$$

as $n \rightarrow \infty$.

Proof. For every $n \in \mathbf{N}$, by the third equality in (3.1) for $f_{\lambda}$ evaluated at $z=\lambda$, we have

$$
\frac{\log \left|\left(f_{\lambda}^{n}\right)^{\prime}(\lambda)\right|}{d^{n}-1}-\frac{n \log d}{d^{n}-1}=\frac{d-1}{d^{n}-1} \sum_{j=0}^{n-1} \log \left|f_{\lambda}^{j}(\lambda)\right|=\frac{d-1}{d^{n}-1} \sum_{j=0}^{n-1} \log \left|f_{\lambda}^{j+1}(0)\right|,
$$

so that

$$
\begin{aligned}
& \int_{\mathbf{P}^{1}}\left|\frac{\log \left|\left(f_{\lambda}^{n}\right)^{\prime}(\lambda)\right|}{d^{n}-1}-g_{H_{\infty}}(\lambda)\right| \mathrm{d} \omega(\lambda) \\
& \leq \frac{d-1}{d^{n}-1} \sum_{j=0}^{n-1} \int_{\mathbf{P}^{1}}|\log | f_{\lambda}^{j+1}(0)\left|-d^{j} \cdot g_{H_{\infty}}(\lambda)\right| \mathrm{d} \omega(\lambda)+\frac{n \log d}{d^{n}-1} \\
& =O\left(n^{2} d^{-n}\right) \text { as } n \rightarrow \infty
\end{aligned}
$$

since by Gauthier-Vigny [9, $§ 4.3$, Proof of Theorem A], we have

$$
\int_{\mathbf{P}^{1}}|\log | f_{\lambda}^{n+1}(0)\left|-d^{n} \cdot g_{H_{\infty}}(\lambda)\right| \mathrm{d} \omega(\lambda)=O(n)
$$

as $n \rightarrow \infty$. This with Lemma 5.1 and $0 \leq \log (1 /[w, \infty])-\log \max \{1,|w|\} \leq \log \sqrt{2}$ on $\mathbf{C}$ completes the proof.

Lemma 5.3. For every $\eta>1$, the Valiron exceptional set

$$
E_{V}\left(\left(\left(f_{\lambda}^{n}\right)^{\prime}(\lambda)\right),\left(\eta^{n}\right)\right):=\left\{a \in \mathbf{P}^{1}: \limsup _{n \rightarrow \infty} \frac{1}{\eta^{n}} \int_{\mathbf{P}^{1}} \log \frac{1}{\left[\left(f_{\lambda}^{n}\right)^{\prime}(\lambda), a\right]} \mathrm{d} \omega(\lambda)>0\right\}
$$

of the sequence $\left(\left(f_{\lambda}^{n}\right)^{\prime}(\lambda)\right)$ in $\mathbf{C}[\lambda]$ with respect to the sequence $\left(\eta^{n}\right)$ in $\mathbf{R}_{>0}$ is a polar subset in $\mathbf{P}^{1}$.

Proof. This is an application of Russakovskii-Shiffman [18, Proposition 6.2] to the sequence $\left(\left(f_{\lambda}^{n}\right)^{\prime}(\lambda)\right)$ in $\mathbf{C}[\lambda]$ since $\sum_{n \in \mathbf{N}} 1 / \eta^{n}<\infty$ for every $\eta>1$.

For every $\eta>1$, every $a \in \mathbf{C} \backslash E_{V}\left(\left(\left(f_{\lambda}^{n}\right)^{\prime}(\lambda)\right),\left(\eta^{n}\right)\right)$, and every $C^{2}$-test function $\phi$ on $\mathbf{P}^{1}$, by Lemmas 5.2 and 5.3, we have

$$
\begin{aligned}
& \left|\int_{\mathbf{P}^{1}} \phi(\lambda) \mathrm{d}\left(\frac{\left(\left(f_{\lambda}^{n}\right)^{\prime}(\lambda)\right)^{*} \delta_{a}}{d^{n}-1}-\mu_{C_{d}}\right)(\lambda)\right| \\
& =\left|\int_{\mathbf{P}^{1}} \phi(\lambda) \mathrm{dd}^{c}\left(\frac{\log \left[\left(f_{\lambda}^{n}\right)^{\prime}(\lambda), a\right]}{d^{n}-1}+\frac{\log \left(1 /\left[\left(f_{\lambda}^{n}\right)^{\prime}(\lambda), \infty\right]\right)}{d^{n}-1}-g_{H_{\infty}}(\lambda)\right)\right| \\
& \leq\left(\sup _{\mathbf{P}^{1}}\left|\frac{\mathrm{dd}^{c} \phi}{\mathrm{d} \omega}\right|\right)\left(\frac{1}{d^{n}-1} \int_{\mathbf{P}^{1}} \log \frac{1}{\left[\left(f_{\lambda}^{n}\right)^{\prime}(\lambda), a\right]} \mathrm{d} \omega(\lambda)\right. \\
& \left.\quad+\int_{\mathbf{P}^{1}}\left|\frac{\log \left(1 /\left[\left(f_{\lambda}^{n}\right)(\lambda), \infty\right]\right)}{d^{n}-1}-g_{H_{\infty}}(\lambda)\right| \mathrm{d} \omega(\lambda)\right) \\
& =o\left((\eta / d)^{n}\right) \text { as } n \rightarrow \infty,
\end{aligned}
$$

where the Radon-Nikodym derivative $\left(\operatorname{dd}^{c} \phi\right) / \mathrm{d} \omega$ on $\mathbf{P}^{1}$ is bounded on $\mathbf{P}^{1}$. 
Acknowledgement. The author thanks the referee for a very careful scrutiny and invaluable comments. This research was partially supported by JSPS Grant-in-Aid for Scientific Research (C), 15K04924.

\section{References}

[1] Azarin, V.S.: Asymptotic behavior of subharmonic functions of finite order. - Mat. Sb. (N.S.) 108(150):2, 1979, 147-167, 303.

[2] Brolin, H.: Invariant sets under iteration of rational functions - Ark. Mat. 6, 1965, $103-144$.

[3] Buff, X.: On the Bieberbach conjecture and holomorphic dynamics. - Proc. Amer. Math. Soc. 131:3, 2003, 755-759.

[4] Dinh, T.-C., and N. Sibony: Equidistribution speed for endomorphisms of projective spaces. - Math. Ann. 347:3, 2010, 613-626.

[5] Doundy, A., and J. H. Hubbard: Itération des polynômes quadratiques complexes. - C. R. Acad. Sci. Paris Sér. I Math. 294:3, 1982, 123-126.

[6] Drasin, D., and Y. Okuyama: Equidistribution and Nevanlinna theory. - Bull. Lond. Math. Soc. 39:4, 2007, 603-613.

[7] ErËmenko, A. E., and M. L. Sodin: Iterations of rational functions and the distribution of the values of Poincaré functions. - Teor. Funktsiǔ Funktsional. Anal. i Prilozhen. 53, 1990, $18-25$.

[8] Freire, A., A. Lopes, and R. MAÑé: An invariant measure for rational maps. - Bol. Soc. Brasil. Mat. 14:1, 1983, 45-62.

[9] Gauthier, T., and G. Vigny: Distribution of postcritically finite polynomials II: Speed of convergence. - arXiv e-prints, 2015.

[10] Gauthier, T., and G. Vigny: Distribution of points with prescribed derivative in polynomial dynamics. - arXiv e-prints, 2016.

[11] Hörmander, L.: The analysis of linear partial differential operators. I. - Grundlehren Math. Wiss. 256, Springer-Verlag, Berlin, 1983.

[12] LJubich, M. J.: Entropy properties of rational endomorphisms of the Riemann sphere. - Ergodic Theory Dynam. Systems 3:3, 1983, 351-385.

[13] Milnor, J.: Dynamics in one complex variable. - Ann. of Math. Stud. 160, Princeton Univ. Press, Princeton, NJ, third edition, 2006.

[14] Nevanlinna, R.: Analytic functions. - Grundlehren Math. Wiss. 162, Springer-Verlag, New York, 1970.

[15] Okuyama, Y.: Valiron, Nevanlinna and Picard exceptional sets of iterations of rational functions. - Proc. Japan Acad. Ser. A Math. Sci. 81:2, 2005, 23-26.

[16] Okuyama, Y.: Nonlinearity of morphisms in non-Archimedean and complex dynamics. Michigan Math. J. 59:3, 2010, 505-515.

[17] Ransford, T.: Potential theory in the complex plane. - Cambridge Univ. Press, Cambridge, 1995.

[18] Russakovskit, A., and B. Shiffman: Value distribution for sequences of rational mappings and complex dynamics. - Indiana Univ. Math. J. 46:3, 1997, 897-932.

[19] Russakovskit, A., and M. Sodin: Equidistribution for sequences of polynomial mappings. Indiana Univ. Math. J. 44:3, 1995, 851-882.

[20] Sodin, M.: Value distribution of sequences of rational functions. - In: Entire and subharmonic functions, Adv. Soviet Math. 11, Amer. Math. Soc., Providence, RI, 1992, 7-20.

[21] Taflin, J.: Equidistribution speed towards the Green current for endomorphisms of $\mathbf{P}^{k}$. Adv. Math. 227:5, 2011, 2059-2081. 
[22] Tsuj,, M.: Potential theory in modern function theory. - Chelsea Publishing Co., New York, reprinting of the 1959 original, 1975.

[23] Yamanoi, K.: Zeros of higher derivatives of meromorphic functions in the complex plane. Proc. Lond. Math. Soc. (3) 106:4, 2013, 703-780.

Received 26 July 2016 • Accepted 23 September 2016 